\title{
Exactly solvable models for tri-atomic molecular Bose-Einstein condensates
}

\author{
G Santos ${ }^{1}$, A Foerster ${ }^{2}$, I Roditi ${ }^{1}$, Z V T Santos ${ }^{1}$ and A P Tonel ${ }^{3}$ \\ ${ }^{1}$ CBPF-Centro Brasileiro de Pesquisas Físicas, Rio de Janeiro RJ, Brazil \\ 2 Instituto de Física da UFRGS, Porto Alegre, RS, Brazil \\ ${ }^{3}$ CCET da Universidade Federal do Pampa/Unipampa, Bagé, RS, Brazil \\ E-mail: gfilho@cbpf.br
}

Received 2 May 2008, in final form 30 May 2008

Published 26 June 2008

Online at stacks.iop.org/JPhysA/41/295003

\begin{abstract}
We construct a family of tri-atomic models for heteronuclear and homonuclear molecular Bose-Einstein condensates. We show that these new generalized models are exactly solvable through the algebraic Bethe ansatz method and derive their corresponding Bethe ansatz equations and energies.
\end{abstract}

PACS numbers: $02.30 . \mathrm{Ik}, 03.65 . \mathrm{Fd}, 03.65 .-\mathrm{w}, 05.30 . \mathrm{Jp}, 03.75 . \mathrm{Nt}$

\section{Introduction}

Since the pivotal experimental achievement that led to realizations of Bose-Einstein condensates (BECs), using ultracold dilute alkali gases [1,2], a great effort has been devoted to the understanding of new properties of BEC. The creation of a molecular BEC compound, which has been obtained by different techniques [3], leads one also to the compelling chemistry of BECs, where the atomic constituents may form molecules, for instance, by Feshbach resonances [4] or photoassociation [5]. These results turned the search for integrable models that could be candidates for describing BEC properties into a very active field of research [6-12]. In fact, exactly solvable models are expected to provide a significant impact in this area, a view that has been promoted in $[13,14]$.

Moreover, the recent experimental evidence for Efimov states in an ultracold cesium gas [15] provided a physical ground for the search of tri-atomic homonuclear molecular BECs. Due to the rapid technological developments in the field of ultracold systems, this experiment is just the beginning of the study of tri-atomic molecules [16, 17].

We introduce, in the present paper, a complete family of new solvable models for both heteronuclear and homonuclear tri-atomic molecular BECs obtained through a combination of two Lax operators constructed using special realizations of the $s u(2)$ and $s u(1,1)$ algebras, as well as a multibosonic representation of $s l(2)$, discussed recently in [18]. 
Until now, integrability was shown for those Hamiltonians describing heteronuclear and homonuclear di-atomic molecular BECs [7]. In the heteronuclear case, two different atoms, labeled $a$ and $b$, can be combined to produce a molecule labeled by $c$. The different degrees of freedom in such models are represented by canonical creation and annihilation operators $\left\{a, b, c, \ldots, a^{\dagger}, b^{\dagger}, c^{\dagger}, \ldots\right\}$ satisfying the usual commutation relations $\left[a, a^{\dagger}\right]=I$, etc, and commuting among different species. The Hamiltonian for the di-atomic heteronuclear model reads $[7,19]$

$$
\begin{aligned}
H=U_{a a} N_{a}^{2}+ & U_{b b} N_{b}^{2}+U_{c c} N_{c}^{2}+U_{a b} N_{a} N_{b}+U_{a c} N_{a} N_{c}+U_{b c} N_{b} N_{c} \\
& +\mu_{a} N_{a}+\mu_{b} N_{b}+\mu_{c} N_{c}+\Omega\left(a^{\dagger} b^{\dagger} c+c^{\dagger} b a\right) .
\end{aligned}
$$

The parameters $U_{i j}$ describe $\mathrm{S}$-wave scattering, $\mu_{i}$ are external potentials and $\Omega$ is the amplitude for interconversion of atoms and molecules. In the homonuclear case, two identical atoms labeled $a$ are combined to produce a molecule $c$ [7]. The generalization to the tri-atomic case is not immediate and in particular, for the homonuclear case, a one-mode multibosonic realization of $s l(2)$ is essential.

In what follows, we will show that it is possible to find other integrable generalized molecular BEC models, more specifically, heteronuclear and homonuclear tri-atomic models. We first introduce two models, one with two identical species of atoms and a different one and another model where there are three different species of atoms. In both cases the three atoms can be combined to form a molecule. We then introduce a model consisting of three identical species of atoms, which uses a recently defined multibosonic realization of the $\operatorname{sl}(2)$ algebra [18]. We present how these generalized models can be derived from a transfer matrix, thus allowing us to show their integrability by the algebraic Bethe ansatz method. We also obtain their corresponding energies and Bethe ansatz equations.

\section{Tri-atomic molecular models}

We are going to introduce in this section three different Hamiltonians describing tri-atomic molecular BECs, two for the hetero-atomic case and one for the homo-atomic case. We are considering the coupling parameters real, such that the Hamiltonians are Hermitian. The $U$ parameters describe the $S$-wave scattering, the $\mu$ parameters are the external potentials and $\Omega$ is the amplitude for interconversion of atoms and molecules. The operators $N_{j}, j=a, b, c, d$, are the number operators acting in the Fock space.

\subsection{Heteronuclear molecular models}

We can construct two models for hetero-atomic molecular BECs, one with two identical species of atoms and a different one and another model where there are three different species of atoms.

2.1.1. Model for two atoms $a$ and one atom $b$. The Hamiltonian that describes the interconversion of a heterogeneous tri-atomic molecule labeled by $c$ with two atoms of type $a$ and one atom of type $b$ is given by

$$
\begin{aligned}
H=U_{a a} N_{a}^{2}+ & U_{b b} N_{b}^{2}+U_{c c} N_{c}^{2}+U_{a b} N_{a} N_{b}+U_{a c} N_{a} N_{c}+U_{b c} N_{b} N_{c} \\
& +\mu_{a} N_{a}+\mu_{b} N_{b}+\mu_{c} N_{c}+\Omega\left(a^{\dagger} a^{\dagger} b^{\dagger} c+c^{\dagger} b a a\right) .
\end{aligned}
$$

Hamiltonian (2) has two independent conserved quantities,

$$
I_{1}=N_{a}+2 N_{c}, \quad I_{2}=N_{a}-2 N_{b},
$$


where $I_{2}$ is the imbalance between the number of atoms of types $a$ and $b$. The total number of particles $N=N_{a}+N_{b}+3 N_{c}$ can be written using these conserved quantities,

$$
N=\frac{3 I_{1}-I_{2}}{2}
$$

and it is also conserved.

Writing the $S$-wave diagonal part of (2) as a combination of the conserved quantities (3) we find

$$
\alpha I_{1}^{2}+\beta I_{2}^{2}+\gamma I_{1} I_{2}
$$

where we have used the following identification for the coupling constants:

$$
\begin{array}{lll}
U_{a a}=\alpha+\beta+\gamma, & U_{b b}=4 \beta, & U_{c c}=4 \alpha, \\
U_{a b}=-4 \beta-2 \gamma, & U_{a c}=4 \alpha+2 \gamma, & U_{b c}=-4 \gamma .
\end{array}
$$

Therefore Hamiltonian (2) can also be written as

$$
H=\alpha I_{1}^{2}+\beta I_{2}^{2}+\gamma I_{1} I_{2}+\mu_{a} N_{a}+\mu_{b} N_{b}+\mu_{c} N_{c}+\Omega\left(a^{\dagger} a^{\dagger} b^{\dagger} c+c^{\dagger} b a a\right) .
$$

This form will be used later when we show how Hamiltonian (2) can be derived from a transfer matrix to establish integrability in the general context of the Yang-Baxter algebra.

2.1.2. Model for three different atoms: $a, b$ and $c$. The Hamiltonian for a model of heterogeneous tri-atomic molecule labeled by $d$ with one atom of type $a$, one atom of type $b$ and one atom of type $c$ is given by

$$
\begin{aligned}
H=U_{a a} N_{a}^{2}+ & U_{b b} N_{b}^{2}+U_{c c} N_{c}^{2}+U_{d d} N_{d}^{2} \\
& +U_{a b} N_{a} N_{b}+U_{a c} N_{a} N_{c}+U_{a d} N_{a} N_{d}+U_{b c} N_{b} N_{c}+U_{b d} N_{b} N_{d}+U_{c d} N_{c} N_{d} \\
& +\mu_{a} N_{a}+\mu_{b} N_{b}+\mu_{c} N_{c}+\mu_{d} N_{d}+\Omega\left(a^{\dagger} b^{\dagger} c^{\dagger} d+d^{\dagger} c b a\right) .
\end{aligned}
$$

Hamiltonian (5) has three independent conserved quantities,

$$
I_{1}=N_{a}+N_{d}, \quad I_{2}=N_{b}+N_{d}, \quad I_{3}=N_{c}+N_{d} .
$$

The physical quantities representing the different imbalances between the number of atoms of different species $\left(J_{a b}, J_{a c}, J_{b c}\right)$ and the total number of atoms $N$ can be expressed as a combination of these quantities, for example,

$$
\begin{aligned}
& J_{a b}=N_{a}-N_{b}=I_{1}-I_{2}, \\
& J_{a c}=N_{a}-N_{c}=I_{1}-I_{3}, \\
& J_{b c}=N_{b}-N_{c}=I_{2}-I_{3}, \\
& N=N_{a}+N_{b}+N_{c}+3 N_{d}=I_{1}+I_{2}+I_{3}
\end{aligned}
$$

and are conserved as well.

We can write the $S$-wave diagonal part of the Hamiltonian (5) in terms of a combination of the independent conserved quantities (6) as

$$
\alpha I_{1}^{2}+\beta I_{2}^{2}+\delta I_{3}^{2}+\gamma I_{1} I_{2}+\rho I_{1} I_{3}+\theta I_{2} I_{3},
$$

where the following identification has been made for the coupling constants

$$
\begin{aligned}
& U_{a a}=\alpha, \quad U_{b b}=\beta, \quad U_{c c}=\delta, \quad U_{d d}=\alpha+\beta+\delta+\gamma+\rho+\theta, \\
& U_{a b}=\gamma, \quad U_{a c}=\rho, \quad U_{b c}=\theta, \quad U_{b d}=2 \beta+\gamma+\theta, \quad U_{c d}=2 \delta+\rho+\theta \text {. }
\end{aligned}
$$

Then Hamiltonian (5) can also be written as

$$
\begin{aligned}
H=\alpha I_{1}^{2}+\beta I_{2}^{2} & +\delta I_{3}^{2}+\gamma I_{1} I_{2}+\rho I_{1} I_{3}+\theta I_{2} I_{3} \\
& +\mu_{a} N_{a}+\mu_{b} N_{b}+\mu_{c} N_{c}+\mu_{d} N_{d}+\Omega\left(a^{\dagger} b^{\dagger} c^{\dagger} d+d^{\dagger} c b a\right) .
\end{aligned}
$$




\subsection{Homonuclear-molecular model}

In the homogeneous case, we can construct a model describing a tri-atomic molecular BEC, where all atoms are identical.

2.2.1. Model for three atoms $a$. The Hamiltonian that describes the interconversion of a homogeneous tri-atomic molecule labeled by $b$ with three atoms of type $a$ is given by

$H=U_{a a} N_{a}^{2}+U_{b b} N_{b}^{2}+U_{a b} N_{a} N_{b}+\mu_{a} N_{a}+\mu_{b} N_{b}$

$$
+\Omega\left(a^{\dagger} a^{\dagger} a^{\dagger} b \alpha_{-}\left(N_{a}\right)+\alpha_{-}\left(N_{a}\right) b^{\dagger} a a a\right)
$$

where $\alpha_{-}\left(N_{a}\right)$ is a function of $N_{a}$ (see the next section for more details) that controls the amplitude of interconversion $\Omega$. This indicates that the density of atoms $N_{a}$ has some influence in the generation of a bound state composed by three identical atoms.

Hamiltonian (9) has one conserved quantity,

$$
I=N_{a}+3 N_{b}
$$

the total number of particles $N=N_{a}+3 N_{b}$.

\section{Integrability and the exact Bethe ansatz solution}

In this section we will discuss the derivation, the integrability and the Bethe ansatz solution of these models. We begin with the $s u(2)$-invariant $R$-matrix, depending on the spectral parameter $u$ :

$$
R(u)=\left(\begin{array}{cccc}
1 & 0 & 0 & 0 \\
0 & b(u) & c(u) & 0 \\
0 & c(u) & b(u) & 0 \\
0 & 0 & 0 & 1
\end{array}\right)
$$

with $b(u)=u /(u+\eta)$ and $c(u)=\eta /(u+\eta)$. Above, $\eta$ is an arbitrary parameter, to be chosen later. It is easy to check that $R(u)$ satisfies the Yang-Baxter equation

$$
R_{12}(u-v) R_{13}(u) R_{23}(v)=R_{23}(v) R_{13}(u) R_{12}(u-v) .
$$

Here $R_{j k}(u)$ denotes the matrix acting non-trivially on the $j$ th and the $k$ th spaces and as the identity on the remaining space.

Next we define the Yang-Baxter algebra $T(u)$,

$$
T(u)=\left(\begin{array}{ll}
A(u) & B(u) \\
C(u) & D(u)
\end{array}\right)
$$

subject to the constraint

$$
R_{12}(u-v) T_{1}(u) T_{2}(v)=T_{2}(v) T_{1}(u) R_{12}(u-v) .
$$

In what follows, we will choose different realizations for the monodromy matrix $\pi(T(u))=$ $L(u)$ to obtain tri-atomic models for heteronuclear and homonuclear molecular BECs. In this construction, the Lax operators $L(u)$ have to satisfy the relation

$$
R_{12}(u-v) L_{1}(u) L_{2}(v)=L_{2}(v) L_{1}(u) R_{12}(u-v) .
$$

Then, defining the transfer matrix, as usual, through

$$
t(u)=\operatorname{tr} \pi(T(u))=\pi(A(u)+D(u)),
$$

it follows from (11) that the transfer matrix commutes for different values of the spectral parameters; i.e., $[t(u), t(v)]=0$. Consequently, the models derived from this transfer matrix will be integrable. Let us now particularize this construction for the hetero and homo-atomic molecular cases. 


\subsection{Heteronuclear-molecular models}

We may choose the following realization for the two models of heteroatomic molecular BECs,

$$
\pi(T(u))=L(u)=u^{-} G L^{S}\left(u^{-}\right) L^{K}\left(u^{+}\right),
$$

with $u^{ \pm}=u \pm \omega, G=\operatorname{diag}(+,-)$, the Lax operator $L^{S}(u)$,

$$
L^{S}(u)=\frac{1}{u}\left(\begin{array}{cc}
u-\eta S^{z} & -\eta S^{+} \\
-\eta S^{-} & u+\eta S^{z}
\end{array}\right)
$$

in terms of the $s u(2)$ Lie algebra with generators $S^{z}$ and $S^{ \pm}$subject to the commutation relations

$$
\left[S^{z}, S^{ \pm}\right]= \pm S^{ \pm}, \quad\left[S^{+}, S^{-}\right]=2 S^{z},
$$

and the Lax operator $L^{K}$,

$$
L^{K}(u)=\frac{1}{u}\left(\begin{array}{cc}
u+\eta K^{z} & \eta K^{-} \\
-\eta K^{+} & u-\eta K^{z}
\end{array}\right)
$$

in terms of the $s u(1,1)$ Lie algebra with generators $K^{z}$ and $K^{ \pm}$subject to the commutation relations

$$
\left[K^{z}, K^{ \pm}\right]= \pm K^{ \pm}, \quad\left[K^{+}, K^{-}\right]=-2 K^{z} .
$$

Now, using two different realizations for the $s u(2)$ and $s u(1,1)$ algebras, that allow different species in the same Lax operator, we will show how to construct the hetero-atomic Hamiltonians (2) and (5) from the transfer matrix (13) and present their exact Bethe ansatz solution.

3.1.1. Model for two atoms $a$ and one atom $b$. Using the following realizations for the $s u(2)$ and $s u(1,1)$ algebras,

$$
\begin{array}{lll}
S^{+}=b^{\dagger} c, & S^{-}=c^{\dagger} b, & S^{z}=\frac{N_{b}-N_{c}}{2}, \\
K^{+}=\frac{\left(a^{\dagger}\right)^{2}}{2}, & K^{-}=\frac{(a)^{2}}{2}, & K^{z}=\frac{2 N_{a}+1}{4},
\end{array}
$$

it is straightforward to check that the Hamiltonian (4) is related to the transfer matrix $t(u)(13)$ through

$$
H=t(u)-\frac{1}{2} u^{-} \eta+\alpha I_{1}^{2}+\beta I_{2}^{2}+\delta I_{1} I_{2},
$$

where the following identification has been made for the parameters,

$$
\mu_{a}=u^{-} \eta, \quad \mu_{c}=-\mu_{b}=u^{+} \eta, \quad \Omega=\frac{\eta^{2}}{2} .
$$

We can apply the algebraic Bethe ansatz method, using as the pseudovacuum the product state $(|0\rangle=|k\rangle \otimes|\phi\rangle$, with $|k\rangle$ denoting the lowest weight state of the $\operatorname{su}(1,1)$ algebra with weight $k$, i.e., $K^{z}|k\rangle=k|k\rangle$ and $|\phi\rangle$ denoting the highest weight state of the $s u(2)$ algebra with weight $m_{z}$ ), to find the Bethe ansatz equations (BAE)

$$
-\frac{\left(v_{i}-\omega-\eta m_{z}\right)\left(v_{i}+\omega+\eta k\right)}{\left(v_{i}-\omega+\eta m_{z}\right)\left(v_{i}+\omega-\eta k\right)}=\prod_{i \neq j}^{M} \frac{v_{i}-v_{j}-\eta}{v_{i}-v_{j}+\eta}, \quad i, j=1, \ldots, M,
$$


and the energies of the Hamiltonian (2) (see, for example, [7, 8])

$$
\begin{aligned}
E= & \left(u^{-}-\eta m_{z}\right)\left(u^{+}+\eta k\right) \prod_{i=1}^{M} \frac{u-v_{i}+\eta}{u-v_{i}} \\
& -\left(u^{-}+\eta m_{z}\right)\left(u^{+}-\eta k\right) \prod_{i=1}^{M} \frac{u-v_{i}-\eta}{u-v_{i}} \\
& -\frac{1}{2} u^{-} \eta+\alpha I_{1}^{2}+\beta I_{2}^{2}+\gamma I_{1} I_{2} .
\end{aligned}
$$

3.1.2. Model for three different atoms: $a, b$ and $c$. Using the following realizations for the $s u(2)$ and $s u(1,1)$ algebras,

$$
\begin{array}{lll}
S^{+}=c^{\dagger} d, & S^{-}=d^{\dagger} c, & S^{z}=\frac{N_{c}-N_{d}}{2}, \\
K^{+}=\frac{\left(a^{\dagger} b^{\dagger}\right)}{2}, & K^{-}=\frac{(a b)}{2}, & K^{z}=\frac{N_{a}+N_{b}+1}{2},
\end{array}
$$

it is straightforward to check that the Hamiltonian (8) is related to the transfer matrix $t(u)(13)$ through

$$
H(u)=t(u)-u^{-} \eta+\alpha I_{1}^{2}+\beta I_{2}^{2}+\delta I_{3}^{2}+\gamma I_{1} I_{2}+\rho I_{1} I_{3}+\theta I_{2} I_{3},
$$

with the following identification for the parameters,

$$
\mu_{a}=\mu_{b}=u^{-} \eta, \quad \mu_{c}=-\mu_{d}=-u^{+} \eta, \quad \Omega=\eta^{2} .
$$

Applying the algebraic Bethe ansatz method we find the Bethe ansatz equations (BAE)

$$
-\frac{\left(v_{i}-\omega-\eta m_{z}\right)\left(v_{i}+\omega+\eta k\right)}{\left(v_{i}-\omega+\eta m_{z}\right)\left(v_{i}+\omega-\eta k\right)}=\prod_{i \neq j}^{M} \frac{v_{i}-v_{j}-\eta}{v_{i}-v_{j}+\eta}, \quad i, j=1, \ldots, M,
$$

and the energies of the Hamiltonian (5)

$$
\begin{aligned}
E= & \left(u^{-}-\eta m_{z}\right)\left(u^{+}+\eta k\right) \prod_{i=1}^{M} \frac{u-v_{i}+\eta}{u-v_{i}} \\
& -\left(u^{-}+\eta m_{z}\right)\left(u^{+}-\eta k\right) \prod_{i=1}^{M} \frac{u-v_{i}-\eta}{u-v_{i}} \\
& -u^{-} \eta+\alpha I_{1}^{2}+\beta I_{2}^{2}+\delta I_{3}^{2}+\gamma I_{1} I_{2}+\rho I_{1} I_{3}+\theta I_{2} I_{3} .
\end{aligned}
$$

\subsection{Homonuclear-molecular model}

In this case we may choose the following realization for the Yang-Baxter algebra

$$
\pi(T(u))=L(u)=\eta^{-1} G L^{b}\left(u-\delta-\eta^{-1}\right) L^{A}(u+\omega),
$$

with $G=\operatorname{diag}(-,+)$ the Lax operator $L^{b}(u)$,

$$
L^{b}(u)=\left(\begin{array}{cc}
u+\eta N_{b} & b \\
b^{\dagger} & \eta^{-1}
\end{array}\right)
$$

in terms of the canonical boson operators $b$ and $b^{\dagger}$ with $N_{b}=b^{\dagger} b$, subject to the commutation relations

$$
[b, b]=\left[b^{\dagger}, b^{\dagger}\right]=0, \quad\left[b, b^{\dagger}\right]=I,
$$

6 
and the Lax operator $L^{A}$,

$$
L^{A}(u)=\left(\begin{array}{cc}
u+\frac{\eta}{2} A_{0} & \eta A_{-} \\
-\eta A_{+} & u-\frac{\eta}{2} A_{0}
\end{array}\right),
$$

in terms of the $s l(2)$ Lie algebra with generators $A_{0}$ and $A_{ \pm}$, subject to the commutation relations

$$
\left[A_{-}, A_{+}\right]=A_{0}, \quad\left[A_{0}, A_{ \pm}\right]= \pm 2 A_{ \pm} .
$$

We find from the $L$ operator (20) the following transfer matrix,

$$
\begin{array}{r}
t(u)=-\eta^{-1}\left(u+\omega+\frac{\eta}{2} A_{0}\right)\left(u-\delta-\eta^{-1}+\eta N_{b}\right) \\
+\eta^{-2}\left(u+\omega-\frac{\eta}{2} A_{0}\right)+b A_{+}+b^{\dagger} A_{-},
\end{array}
$$

with

$$
t(0)=\eta^{-1} \omega\left(\delta+2 \eta^{-1}\right)-\omega N_{b}+\frac{1}{2}\left(\delta-\eta N_{b}\right) A_{0}+b A_{+}+b^{\dagger} A_{-} .
$$

There is a one-mode realization of the $s l(2)$ algebra [18]

$$
A_{0}=\alpha_{0}(N), \quad A_{-}=\alpha_{-}(N) a^{l}, \quad A_{+}=\left(a^{\dagger}\right)^{l} \alpha_{-}(N)
$$

with

$$
\begin{aligned}
& \alpha_{0}(N)=\frac{2}{l}(N-R)+\alpha_{0}(R) \\
& \alpha_{-}(N)=\sqrt{\frac{N !}{(N+l) !}\left(\frac{1}{l}(N-R)+\alpha_{0}(R)\right)\left(\frac{1}{l}(N-R)+1\right)}
\end{aligned}
$$

where $N=a^{\dagger} a$ and $l \in \mathbb{N}$. The operator $R$ is

$$
R= \begin{cases}0, & \text { if } \quad l=1 \\ \frac{l-1}{2}+\sum_{m=1}^{l-1} \frac{e^{-(2 \pi m / l) N}}{e^{(2 \pi m / l)}-1}, & \text { if } \quad l>1\end{cases}
$$

and acts on the states $\{|n\rangle\}$ as $R|n\rangle=n \bmod l|n\rangle$. The function $\alpha_{0}(R)$ is a function of the spectrum of $R$. For $n=r<l$, we have

$$
\frac{1}{l}(N-R)|r\rangle=0|r\rangle
$$

with $A_{0}=\alpha_{0}(R)$ such that $\alpha_{0}(R)|r\rangle=\alpha_{0}(r)|r\rangle$ and $R|r\rangle=r|r\rangle$. The operator $R$ commutes with $a^{l}$ and $\left(a^{\dagger}\right)^{l}$ and so is a conserved quantity for all models presented in this paper.

Now we will apply this realization to show how to construct Hamiltonian (9) from the transfer matrix (22) and present their exact Bethe ansatz solution. Note that direct combinations of $s l(2)$ and $s u(1,1)$ as in the heteronuclear cases do not lead to suitable Lax operators. However, through the present realization we are able to build a viable Lax operator for the homonuclear-molecular model.

For $l=3$, which is the case of interest here, it is straightforward to check that Hamiltonian (9) is related to the transfer matrix $t(0)$ (23) through

$$
H=t(0)
$$


where we have the following identification,

$$
\begin{aligned}
& \eta=9 U_{a}+U_{b}-3 U_{a b} \\
& 6(\omega+\delta)=\left(18 U_{a}-2 U_{b}\right) N-\eta \rho+18 \mu_{a}-6 \mu_{b} \\
& 36 \eta^{-1} \omega \delta+72 \omega \eta^{-2}=\eta \rho^{2}+\left[\left(4 U_{b}-6 U_{a b}\right) N+6\left(\mu_{b}-3 \mu_{a}+\omega\right)\right] \rho \\
& +4 U_{b} N^{2}+12\left(\mu_{b}+\omega\right) N
\end{aligned}
$$

with $\rho \equiv \rho(R)=3 \alpha_{0}(R)-2 R$. Let $|0\rangle_{b}$ denote the Fock vacuum state and let $|r\rangle_{A}$ denote the lowest weight state of the $\operatorname{sl}(2)$ algebra where $r=0,1,2$, are the eingenvalues of $R$ for $l=3$ and $N=n l+r$, with $n \in N$. On the product state $|\Psi\rangle=|r\rangle_{A} \otimes|0\rangle_{b}$ we can apply the algebraic Bethe ansatz method for finding the Bethe ansatz equations (BAE)

$$
\frac{\left(1-\eta v_{i}+\eta \delta\right)\left(v_{i}+\omega+\frac{\eta}{2} \alpha_{0}(r)\right)}{v_{i}+\omega-\frac{\eta}{2} \alpha_{0}(r)}=\prod_{i \neq j}^{M} \frac{v_{i}-v_{j}-\eta}{v_{i}-v_{j}+\eta}, \quad i, j=1, \ldots, M,
$$

and the energies of the Hamiltonian (9)

$$
E=\eta^{-1}\left(\delta+\eta^{-1}\right)\left(\omega+\frac{\eta}{2} \alpha_{0}(r)\right) \prod_{i=1}^{M} \frac{v_{i}-\eta}{v_{i}}+\eta^{-2}\left(\omega-\frac{\eta}{2} \alpha_{0}(r)\right) \prod_{i=1}^{M} \frac{v_{i}+\eta}{v_{i}}
$$

\section{Summary}

We have introduced three new integrable models for both homogeneous and heterogeneous tri-atomic molecular BECs obtained through a combination of Lax operators constructed using special realizations of the $s u(2)$ and $s u(1,1)$ algebras and a particular one-mode multibosonic representation of $s l(2)$, possibilities that were overlooked in previous studies. The models were solved by means of the algebraic Bethe ansatz method and their corresponding energies and Bethe ansatz equations were derived. We hope that our models may be of physical relevance as long as new experiments are performed in the field, which is just in its initial stage.

\section{Acknowledgments}

A F thanks J Links for useful discussions. I R acknowledges FAPERJ (Fundação de Amparo à Pesquisa do Estado do Rio de Janeiro). The authors also acknowledge support from CNPq (Conselho Nacional de Desenvolvimento Científico e Tecnológico).

\section{References}

[1] Cornell E A and Wieman C E 2002 Rev. Mod. Phys. 74875

[2] Anglin J R and Ketterle W 2002 Nature 416211

[3] Zoller P 2002 Nature 417493

[4] Papp S B and Wieman C E 2006 Preprint cond-mat/0607667

[5] Damski B, Santos L, Tiemann E, Lewenstein M, Kotochigova S, Julienne P and Zoller P 2003 Phys. Rev. Lett. 90110401

[6] Zhou H-Q, Links J, Gould M and McKenzie R 2003 J. Math. Phys. 444690

[7] Links J, Zhou H-Q, McKenzie R H and Gould M D 2003 J. Phys. A: Math. Gen. 36 R63

Zhou H-Q, Links J, McKenzie R H and Guan X-W 2003 J. Phys. A: Math. Gen. 36 L113

[8] Foerster A, Links J and Zhou H-Q 2003 Classical and Quantum Nonlinear Integrable Systems: Theory and Applications ed A Kundu (Bristol: Institute of Physics Publishing) pp 208-233

[9] Dukelsky J, Dussel G, Esebbag C and Pittel S 2004 Phys. Rev. Lett. 93050403

[10] Ortiz G, Somma R, Dukelsky J and Rombouls S 2005 Nucl. Phys. B 707421 
[11] Kundu A 2007 Theor. Math. Phys. 151831

[12] Foerster A and Ragoucy E 2007 Nucl. Phys. B 777373

[13] Héritier M 2001 Nature 41431

[14] Batchelor M T 2007 Phys. Today 6036

[15] Kraemer T et al 2006 Nature 440315

[16] Braaten E and Hammer H-W 2007 Ann. Phys., NY 322120

[17] Esry B D and Greene C 2006 Nature 440289

[18] Goliński T, Horowski M, Odzijewicz A and Sliżewska A 2007 J. Math. Phys. 48023508

[19] Duncan M, Foerster A, Links J, Mattei E, Oelkers N and Tonel A 2007 Nucl. Phys. B 767227 